\title{
Inventário de vespas (Hymenoptera, Vespidae) no Mato Grosso do Sul, Brasil
}

\author{
Tiago Henrique Auko ${ }^{1,2}$, Vander Carbonari ${ }^{2}$, Daniele Guedes Ribeiro ${ }^{1,2}$ \& Rogério Silvestre ${ }^{1,2}$
}

\author{
1. Programa de Pós-Graduação em Entomologia e Conservação da Biodiversidade, Faculdade de Ciências Biológicas e Ambientais, Universidade Federal da \\ Grande Dourados (UFGD), Brasil. (henriqueauko@yahoo.com.br) \\ 2. Laboratório de Ecologia de Hymenoptera - Hecolab, Universidade Federal da Grande Dourados (UFGD), Brasil.
}

Recebido 22 novembro 2016

Aceito 6 fevereiro 2017

DOI: $10.1590 / 1678-4766 e 2017125$

\begin{abstract}
Checklist of wasp (Hymenoptera, Vespidae) from Mato Grosso do Sul, Brazil. This is a preliminary survey of wasps (Vespidae) conducted in a representative points of the biomes from Mato Grosso do Sul: Pantanal, Chaco, Cerrado and Atlantic Forest, covering periods of sampling between 2006 to 2011, being the first effort to quantify the Vespidae fauna for this State. The samples were concentrated primarily in areas with good conservation status, as Parque Nacional da Serra da Bodoquena, Reserva Particular do Patrimônio Natural Eng. Eliezer Batista (RPPNEEB), Maciço do Urucum, riparian forests of the rivers Aporé and Sucuriú, Atlantic Forest fragments of Dourados municipality and Brazilian Chaco. The sampling techniques employed were: active search, with entomological nets and Malaise and Möerick traps. The methods were not standardized for the locations. In total 105 species in 35 genera of Vespidae were recorded for Mato Grosso do Sul. The genera with high richness were: Omicron and Zethus, with 10 species each one. Among the solitary wasps, Pachodynerus guadulpensis was the most abundant, with 32 individuals. To the social wasps Agelaia multipicta, Polybia ignobilis, and Polybia (gr. occidentalis) sp. were the most abundant. Trimeria rubra (Masarinae) was sampled for the first time in Mato Grosso do Sul State. The knowledge status of biodiversity is fundamental for future strategies of management's plans, resulting in the selection of priority areas for conservation.
\end{abstract}

KEYWORDS. Polistinae, Eumeninae, Masarinae, Serra da Bodoquena, Biota-MS Program.

RESUMO. Este levantamento preliminar de vespas (Vespidae), realizado em pontos representativos dos biomas do Mato Grosso do Sul: Pantanal, Chaco, Cerrado e Mata Atlântica, abrange períodos de coletas entre 2006 a 2011, sendo a primeira tentativa de quantificar a fauna de Vespidae para o estado. A amostragem concentrou-se basicamente em áreas com bom estado de conservação, como o Parque Nacional da Serra da Bodoquena, Reserva Particular do Patrimônio Natural Engenheiro Eliezer Batista (RPPNEEB), Maciço do Urucum, matas ciliares dos rios Aporé e Sucuriú, fragmento de mata Atlântica em Dourados e fitofisionomias do Chaco Brasileiro. As técnicas de coletas empregadas foram: busca ativa, com rede entomológica e com armadilhas de Malaise e Möerick. Os métodos de amostragem não foram padronizados em todas as localidades. No total 105 espécies em 35 gêneros de Vespidae foram registrados para o Mato Grosso do Sul. Os gêneros com maior riqueza foram: Omicron e Zethus, com 10 espécies cada; entre as vespas solitárias, Pachodynerus guadulpensis foi a que obteve o maior número de indivíduos (32). Entre as vespas sociais, Agelaia multipicta, Polybia ignobilis e Polybia (gr. occidentalis) sp. foram as mais abundantes. Trimeria rubra (Masarinae) foi amostrada pela primeira vez no Chaco Brasileiro. O conhecimento da biodiversidade do estado é fundamental para estratégias futuras de planos de manejos, implicando na escolha de áreas prioritárias para a Conservação.

PALAVRAS-CHAVE. Polistinae, Eumeninae, Masarinae, Serra da Bodoquena, Programa Biota-MS.

Os Vespidae, popularmente conhecidos como vespas e marimbondos, são insetos cosmopolitas e distribuemse preferencialmente em áreas tropicais. No mundo são conhecidas 5.000 espécies e 268 gêneros, em seis subfamílias descritas, porém apenas três ocorrem na América do Sul (Brothers \& Finnamore, 1993; Carpenter \& Marques, 2001; PiCKeTT \& CARPENTER, 2010).

Eumeninae, com 3.579 espécies e 210 gêneros registrados no Mundo, apresenta a maior riqueza do grupo, aproximadamente 279 espécies em 32 gêneros foram registradas para o Brasil (WILLINK \& RoIG-AlsinA, 1998; Carpenter \& Garcete-Barrett, 2002; Pickett \& Carpenter, 2010; Auko \& Silvestre, 2015). Polistinae, também cosmopolita, possui 958 espécies e 26 gêneros conhecidos, sendo que 22 destes gêneros e 304 espécies ocorrem no Brasil, com 106 espécies endêmicas no país (CARPENTER \& MARQUES, 2001). Masarinae restringe sua distribuição a áreas secas da Austrália, Sul da África, Mediterrâneo e na Região Neotropical, mas precisamente em áreas andinas, conta com 298 espécies e 14 gêneros catalogadas no mundo (SARMIENTO \& CARPENTER, 2006).

Os vespídeos podem ser encontrados nos mais variados hábitats, ocupando áreas desde o nível do mar até pouco mais de quatro mil metros de altitude (SARMIENTO, 1997). Suas larvas se desenvolvem às custas de proteína animal adquirida pelo adulto, que por sua vez, alimenta-se basicamente de néctar, mas podem consumir também açúcares excretado de pulgões e secreções de cadáveres (O’DonNell, 1995). 
O conhecimento da diversidade de Vespidae no Brasil ainda é bastante reduzido para a maioria dos estados, salvo os trabalhos localizados na Mata Atlântica de São Paulo (Togni, 2009) e da Bahia (SAntos et al., 2007), no Cerrado de São Paulo (Mechi, 1996; Mechi \& Moraes, 2000; Junior \& Noll, 2011), Goiás (SANtos, 1996; Raw, 1998a), Mato Grosso (Diniz \& Kitayama, 1998), Minas Gerais (ElPINO-CAMPOS et al., 2007) e Bahia (SANTOs et al., 2009). Na Floresta Amazônica existem levantamentos no Pará (Silveira, 2002; Silva, 2007; Silva \& SilveIra, 2009), no Amazonas (Silveira et al., 2008), no Acre (SouZa et al., 2003; Morato et al., 2008) e no Amapá (Raw, 1998b). No Pampa as vespas foram estudadas por HeRMES \& KöHLER (2004) e Somavila (2010). Em florestas estacionais temos coletas em São Paulo (Gomes \& NolL, 2009; JunIOR \& NolL, 2011). Para a América do Sul, os inventários de Vespidae são restritos ao Peru (RASMUSSEN \& AsENJo, 2009), Paraguai (Garcete-Barrett, 1996) e Colômbia (Sarmiento, 1997, 1999).

As vespas apresentam uma relação bastante estreita com a matriz fitofisionômica; a composição da comunidade destes insetos pode ser utilizada para a avaliação de algumas variáveis ambientais, indicando a integridade e a complexidade do ecossistema em questão (MorATO, 2004; LOYOLA \& MARTINS, 2008). Este inventário da composição de Vespidae no Mato Grosso do Sul tem como objetivo contribuir para o conhecimento da biota do estado, que é extremamente heterogêneo em termos de hábitats e que vem sofrendo uma acelerada perda de seus ecossistemas naturais.

\section{MATERIAL E MÉTODOS}

Área de estudo. O estado do Mato Grosso do Sul situa-se na porção central da grande área diagonal de formações abertas da América do Sul, que se estende desde a Caatinga no nordeste do Brasil até o Chaco na Argentina, onde ocorrem áreas de contato entre o Pantanal, o Chaco e o Cerrado. Esta diagonal de áreas abertas abriga grande diversidade de ecossistemas, incluindo um elevado número de espécies de flora e fauna endêmicas (Bucher, 1980; Vanzolini, 1988; Zanella, 2011). Dentre os componentes fitofisionômicos que a compõe encontram-se as matas estacionais (deciduais e semideciduais), que exercem um papel chave na distribuição de diversos elementos da Biota (Prado \& Gibbs, 1993; Morrone et al., 2004; Silvestre et al., 2012). No sul do estado encontram-se diversas "manchas" com elementos de Mata Atlântica, estendendo-se até a Serra da Bodoquena (Ротт \& Ротт, 2003).

Este levantamento de vespas realizado no Mato Grosso do Sul é resultado de uma série de projetos independentes, explorando os diferentes ecossistemas: Pantanal, Chaco, Floresta Estacional, Cerrado e Mata Atlântica. As localidades amostradas foram representadas em cinco pontos (Fig. 1). Os pontos amostrados dentro das cinco localidades foram georeferenciados e são indicados na Tab.I.

A Serra da Bodoquena é constituída por um planalto escarpado a oeste, no sentido da planície do Pantanal, ligando-

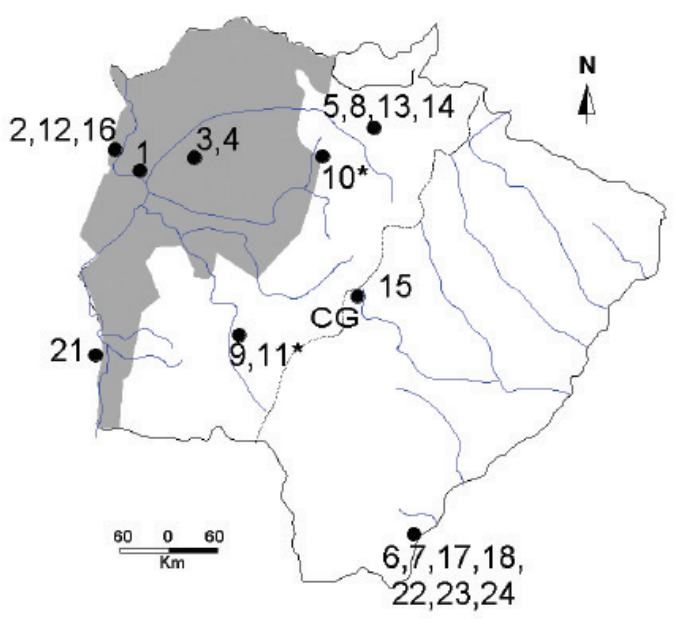

Fig. 1. Localidades amostradas para a fauna de Vespidae no Mato Grosso do Sul, Brasil entre 2006 e 2011.

se à planície de do Rio Miranda. Totaliza cerca de $300 \mathrm{~km}$ de comprimento e 20 a $50 \mathrm{~km}$ de largura, sustentadas por rochas calcárias (BoGGIANI et al., 1993). Apresenta vegetação nativa de Floresta Estacional Decidual e Semidecidual, Cerrado, Cerradão e áreas de encraves. Nos topos dos morros apresenta afloramentos rochosos magmáticos e, em elevações acima de $400 \mathrm{~m}$, surge à formação de Campo Rupestre, com ocorrência de cactáceas. Foram realizadas oito expedições de coletas na Serra da Bodoquena, entre os anos de 2007 a 2011, com dez sítios amostrados.

A região do Aporé-Sucuriú foi amostrada para visitantes florais e as vespas foram encaminhadas ao Laboratório de Ecologia de Hymenoptera da FCBA-UFGD. As coletas foram realizadas na bacia dos Aporé e Sucuriú, envolvendo os municípios de Chapadão do Sul, Inocência e Costa Rica. Este complexo caracteriza-se por apresentar fitofisionomias que vão desde o Campo Limpo ao Cerrado sentido restrito, incluindo brejos e vegetação exótica com eucalipto (Pagotto \& Souza, 2006).

A Mata do Azulão, localizada no sul do estado, no município de Dourados, consiste em um fragmento de Floresta Estacional Semidecidual Atlântica, de aproximadamente 10 ha, com grande atividade agrícola e pecuária na região de entorno.

Duas localidades foram amostradas na região do Pantanal, sendo elas: Reserva Particular do Patrimônio Natural Engenheiro Eliezer Batista e o Maciço do Urucum, ambas localizadas no município de Corumbá, na bacia do Rio Paraguai, com as formações florestais de Floresta Estacional (mata seca), Matas Ciliares e Cordilheiras.

Na região de Porto Murtinho temos a formação florestal de Chaco, com as transições florísticas e ecótonos de diferentes regiões fitoecológicas, sendo reconhecidas as seguintes fitofisionomias: Savana Estépica GramíneoLenhosa, Savana Estépica Arbórea, Parque de Carandazais, formações de encrave Chaco/Floresta Decidual e Chaco/ Cerrado, brejos, banhados e salitres (ABDON \& Silva, 2006; NoGUCHI et al., 2009). 
Tabela I. Pontos de coletas de vespas (Hymenoptera, Vespidae) no período entre 2006 a 2011 no Mato Grosso do Sul, Brasil.

\begin{tabular}{|c|c|c|}
\hline Localidade & Coordenadas & Data \\
\hline \multicolumn{3}{|c|}{ 1. SERRA DA BODOQUENA } \\
\hline Faz. Califórnia & $56^{\circ} 50^{\prime} 57^{\prime \prime} \mathrm{S}, 20^{\circ} 42^{\prime} 11^{\prime \prime} \mathrm{W}$ & II.2007; XI.2009 \\
\hline Faz. Marambaia & $56^{\circ} 47^{\prime} 31^{\prime \prime} \mathrm{S}, 20^{\circ} 50^{\prime} 26^{\prime \prime} \mathrm{W}$ & V.2007 \\
\hline Faz. Santa Laura & $56^{\circ} 44^{\prime} 54^{\prime \prime} \mathrm{S}, 20^{\circ} 47^{\prime} 59^{\prime \prime} \mathrm{W}$ & XI.2007; IV.2008 \\
\hline Faz. Santa Maria & $56^{\circ} 45^{\prime} 48^{\prime \prime} \mathrm{S}, 21^{\circ} 25^{\prime} 39^{\prime \prime} \mathrm{W}$ & II.2008 \\
\hline Faz. Pitangueiras & $56^{\circ} 35^{\prime} 20^{\prime} \mathrm{S}, 20^{\circ} 52^{\prime} 13^{\prime \prime} \mathrm{W}$ & IV.2008; XI.2009 \\
\hline Faz. Campo Verde & $56^{\circ} 45^{\prime} 40^{\prime \prime} \mathrm{S}, 21^{\circ} 22^{\prime} 49^{\prime \prime} \mathrm{W}$ & XII.2008 \\
\hline Rio Taquaral & $56^{\circ} 38^{\prime} 14^{\prime \prime} \mathrm{S}, 21^{\circ} 06^{\prime} 27^{\prime \prime} \mathrm{W}$ & XI.2009 \\
\hline Faz. Boqueirão & $56^{\circ} 43^{\prime} 08^{\prime \prime} \mathrm{S}, 21^{\circ} 07^{\prime} 14^{\prime \prime} \mathrm{W}$ & X.2011 \\
\hline RPPN Cara da Onça & $56^{\circ} 44^{\prime} 13^{\prime \prime} \mathrm{S}, 20^{\circ} 44^{\prime} 24^{\prime \prime} \mathrm{W}$ & XII.2011 \\
\hline Cachoeira do Apa & $57^{\circ} 32^{\prime} 13^{\prime} \mathrm{S}, 22^{\circ} 09^{\prime} 49^{\prime \prime} \mathrm{W}$ & V.2010 \\
\hline 2. APORÉ-SUCURIÚ & $52^{\circ} 30^{\prime} 00^{\prime} \mathrm{S}, 19^{\circ} 13^{\prime} 00^{\prime \prime} \mathrm{W}$ & 2006 \\
\hline 3. DOURADOS & $54^{\circ} 55^{\prime} 05^{\prime} \mathrm{S}, 22^{\circ} 12^{\prime} 24^{\prime \prime} \mathrm{W}$ & $2009 / 2010$ \\
\hline \multicolumn{3}{|l|}{ 4. PANTANAL } \\
\hline Maciço do Urucum & $57^{\circ} 28^{\prime} 00^{\prime} \mathrm{S}, 19^{\circ} 11^{\prime} 00^{\prime \prime} \mathrm{W}$ & V.2011; IX.2011 \\
\hline Serra do Amolar & $57^{\circ} 28^{\prime} 27^{\prime \prime} \mathrm{S}, 18^{\circ} 05^{\prime} 25^{\prime \prime} \mathrm{W}$ & VII.2010; III.2011 \\
\hline \multicolumn{3}{|l|}{ 5. CHACO } \\
\hline Faz. Porto Conceição & $57^{\circ} 54^{\prime} 38^{\prime} \mathrm{S}, 21^{\circ} 29^{\prime} 05^{\prime \prime} \mathrm{W}$ & XI.2010 \\
\hline Faz. Santa Virgínia & $57^{\circ} 53^{\prime} 54^{\prime \prime} \mathrm{S}, 21^{\circ} 43^{\prime} 10^{\prime \prime} \mathrm{W}$ & V.2011 \\
\hline
\end{tabular}

Métodos de coleta. Para este inventário rápido contemplando a diversidade de vespas (Vespidae) no Mato Grosso do Sul, as seguintes técnicas de amostragens foram empregadas de forma não padronizadas:

Coleta ativa. Rede entomológica: nesta metodologia incluem-se a observação em lugares que proporcionam algum tipo de recurso para as vespas, como observações de flores, ranchos abandonados e barrancos de rios. Esta foi a única técnica de coleta utilizada em todas as áreas do Mato Grosso do Sul amostradas neste trabalho.

Coleta passiva. Armadilha de Möericke: usada em coletas realizadas no Chaco e na Serra da Bodoquena. Ao todo foram dispostas 550 bandejas amarelas, contendo água e detergente, sendo 50 por expedição de coleta, distando 10 $\mathrm{m}$ uma armadilha da outra.

Armadilha de Malaise. Esta técnica foi utilizada no Chaco, na Serra da Bodoquena e no Pantanal (RPPN). Ao todo no inventário foram recolhidas 30 amostras, dispostas rente ao solo, por quatro dias consecutivos.

O material foi comparado com os espécimes depositados na coleção referência do Museu de Zoologia da USP e alguns exemplares foram determinados por especialistas de seus respectivos grupos. O material testemunho foi depositado na coleção de Hymenoptera do Museu de Biodiversidade da Faculdade de Ciências Biológicas e Ambientais (MuBio/UFGD), sob curadoria do Prof. Dr. Rogério Silvestre.

\section{RESULTADOS}

Foram amostrados 648 indivíduos, com 105 espécies e 35 gêneros registrados, representando as três subfamílias presentes na Região Neotropical (Tab. II). Eumeninae foi o grupo taxonômico mais rico, com 66 espécies, 21 gêneros e 230 indivíduos capturados. Polistinae, com 409 exemplares capturados é representado pelos Epiponini, com 21 espécies e 10 gêneros, pelos Mischocyttarini com oito espécies e um gênero (Mischcyttarus Saussure) e pelos
Polistini, também com oito espécies e um gênero (Polistes Latreille). Masarinae, com oito registros, foi representado apenas pelos Masarini, com dois gêneros e duas espécies (Ceramiopsis paraguayensis Bertoni, 1921 e Trimeria rubra Hermes \& Melo, 2006).

Os gêneros que apresentaram a maior riqueza entre todos os Vespidae amostrados foram Omicron Saussure e Zethus Fabricius com 10 espécies cada, enquanto que a maior abundância em números de registros foi de Polybia (gr. occidentalis) sp., Agelaia multipicta (Haliday, 1836) e Polybia ignobilis (Haliday, 1836), respectivamente com 84, 64 e 60 registros. Entre as vespas solitárias, Pachodynerus guadulpensis (Saussure, 1853) foi a que obteve o maior número de indivíduos (32).

Foram amostradas 52 espécies na Serra da Bodoquena, 39 no Pantanal, 28 no Chaco, 25 na região do Aporé-Sucuriú e 20 em Dourados. Sessenta e nove espécies foram registradas em apenas uma das localidades amostradas. Quatro espécies - Brachygastra lecheguana (Latreille, 1824), Polybia sericea (Oliver, 1791), P. ignobilis e P. (gr. occidentalis) sp. - estão presentes em todas as áreas amostradas. Agelaia multipicta, Zeta argillaceum (Linnaeus, 1758), Pachodynerus guadulpensis e Hypalastoroides brasiliensis (Saussure, 1856) foram encontradas em quatro das cinco regiões amostradas.

\section{DISCUSSÃO}

Não existem estimativas do número total de vespas para o Brasil, devido à carência de estudos taxonômicos e sistemáticos (Souza et al., 2003). A ocorrência de 105 espécies e de 35 gêneros no Mato Grosso do Sul confirma uma alta diversidade imaginada para este estado, considerando que muitas regiões no estado ainda não foram amostradas, principalmente na bacia do rio Paraná. Segundo SANToS et al. (2007), ambientes estruturalmente mais complexos tendem a apresentar maior riqueza e maior diversidade de espécies de vespas devido à maior oferta de microhábitats, 
Tab. II. Lista das espécies de vespas (Hymenoptera: Vespidae) amostradas no Mato Grosso do Sul de 2006 a 2011 (SB, Serra da Bodoquena; P, Pantanal; Ch, Chaco; AS, Complexo Aporé-Sucuriú; MA, Mata do Azulão).

\begin{tabular}{|c|c|c|c|c|c|}
\hline & & & alida & & \\
\hline Especies & SB & $\mathrm{P}$ & $\mathrm{Ch}$ & AS & MA \\
\hline
\end{tabular}

Vespidae

Eumeninae

Alphamenes sp.

Ancistroceroides atripes (Fox, 1902)

Ancistroceroides alasteroides (Saussure, 1852)

Ancistroceroides conjunctus (Fox, 1902)

Ancistroceroides rufimaculatus (Fox, 1902)

Ancistroceroides venustus (Brethes, 1905)

Ancistroceroides sp.

Antezumia sp.

Brachymenes dyscherus (Saussure, 1852)

Cyphomenes anisitsii (Brethes, 1906)

Eumenes rufomaculatus Fox, 1899

Hypalastoroides brasiliensis (Saussure, 1856)

Hypalastoroides elongatus (Brethes, 1906)

Hypalastoroides nitidus (Brethes, 1906)

Hypalastoroides paraguayensis Zavattari, 1911

Hypancistrocerus advena (Saussure, 1856)

Hypancistrocerus reflectorius (Dalla Torre, 1904)

Hypalastoroides sp.

Minixi brasilianum (Saussure, 1875)

Minixi suffusum (Fox, 1899)

Monobia angulosa Saussure, 1852

Monobia apicalipennis Saussure, 1852

Montezumia azurescens (Spinola, 1851)

Montezumia ferruginea Saussure, 1856

Montezumia infernalis (Spinola, 1851)

Montezumia petiolata de Saussure, 1856

Montezumia sp.

Omicron criticum richardsi Giordani Soika, 1978

Omicron paranymphum (Zavattari, 1912)

Omicron ruficolle schunkei Giordani Soika, 1978

Omicron spegazzinii Brethes, 1905

Omicron tegulare (Fox, 1899)

Omicron tuberculatum (Fox, 1899)

Omicron sp. 1

Omicron sp. 2

Omicron sp. 3

Omicron sp. 4

Pachymenes ghilianii (Spinola, 1851)

Pachymenes picturatus (Fox, 1899)

Pachymenes sp.

Pachodynerus brevithorax (Saussure, 1852)

Pachodynerus corumbae (Fox, 1902)

Pachodynerus grandis Willink \& Roig-Alsina, 1998

Pachodynerus guadulpensis (Saussure, 1853)

Pachodynerus nasidens (Latreille, 1812)

Pachodynerus serrulatus Brèthes, 1920

Parancistrocerus longicornutus (Dalla Torre, 1904)

Parancistrocerus sp. 1

Parancistrocerus sp. 2

Plagiolabra andina Brethes, 1906

Plagiolabra nigra Schulthess, 1903

Santamenes novarae (Saussure, 1867)

Stenodynerus suffusus (Fox, 1902)

Stenodynerus sp.

Stenonartonia apicipennis (Fox, 1902)

Zeta argillaceum (Linnaeus, 1758)

Zethus chapadensis Bohart \& Stange, 1965

Zethus cylindricus Fox, 1899

Zethus dubius Smith, 1857

Zethus iheringi Zavattari, 1912

Zethus hilarianus Saussure, 1856

Zethus mexicanus Bohart \& Stange, 1965

Zethus romandinus Saussure, 1852

\begin{tabular}{|c|c|c|c|c|}
\hline- & - & - & 1 & - \\
\hline 1 & - & - & - & - \\
\hline- & - & 1 & - & - \\
\hline 7 & - & - & - & - \\
\hline- & 1 & 1 & - & - \\
\hline- & - & 1 & - & - \\
\hline- & 2 & - & - & - \\
\hline- & 1 & - & - & - \\
\hline 4 & - & - & - & - \\
\hline- & - & - & - & 3 \\
\hline 1 & 2 & - & - & \\
\hline 6 & 3 & 11 & 1 & - \\
\hline 1 & - & - & - & - \\
\hline- & - & 1 & - & - \\
\hline 2 & 1 & 1 & - & - \\
\hline 1 & - & 2 & - & - \\
\hline- & 1 & - & - & - \\
\hline- & - & 1 & 1 & - \\
\hline 1 & - & - & - & - \\
\hline 2 & 1 & - & - & - \\
\hline 6 & - & - & - & - \\
\hline 2 & - & - & - & - \\
\hline 2 & - & - & - & - \\
\hline 6 & - & 1 & - & - \\
\hline 2 & - & - & - & - \\
\hline 2 & - & - & - & 1 \\
\hline- & - & 3 & - & - \\
\hline- & 1 & - & - & - \\
\hline- & - & - & - & 1 \\
\hline- & 4 & - & 2 & - \\
\hline 2 & - & - & - & - \\
\hline- & 1 & - & - & - \\
\hline 9 & 3 & - & - & - \\
\hline- & 3 & - & - & - \\
\hline- & - & - & 4 & - \\
\hline- & - & - & 2 & - \\
\hline- & 1 & - & - & - \\
\hline 1 & - & - & - & - \\
\hline 2 & 1 & - & - & - \\
\hline- & - & - & 2 & - \\
\hline 3 & - & - & - & - \\
\hline- & - & 1 & - & - \\
\hline 4 & - & - & - & - \\
\hline 17 & 4 & 1 & - & 10 \\
\hline 4 & - & - & - & - \\
\hline- & - & - & - & 2 \\
\hline 3 & - & - & - & - \\
\hline 1 & - & - & - & - \\
\hline- & 1 & - & - & - \\
\hline- & - & 1 & - & - \\
\hline- & - & 1 & - & - \\
\hline 2 & 1 & - & - & - \\
\hline 1 & 1 & - & - & - \\
\hline- & 2 & - & - & - \\
\hline- & 1 & - & - & - \\
\hline 9 & - & 3 & 6 & 1 \\
\hline- & 1 & - & - & - \\
\hline 7 & 3 & - & - & - \\
\hline- & 1 & - & - & 1 \\
\hline- & - & - & 1 & - \\
\hline- & - & - & 1 & - \\
\hline- & - & - & 1 & - \\
\hline- & 1 & 1 & - & - \\
\hline
\end{tabular}


Tab. II. Cont.

\begin{tabular}{|c|c|c|c|c|c|}
\hline \multirow{2}{*}{ Espécies } & \multicolumn{5}{|c|}{ Localidades } \\
\hline & SB & $\mathrm{P}$ & $\mathrm{Ch}$ & AS & MA \\
\hline Zethus schrottkyanus von Ihering, 1911 & - & 1 & - & - & - \\
\hline Zethus sessilis Fox, 1899 & - & 1 & 1 & - & - \\
\hline Zethus (gr. almecos) sp. & - & 1 & - & 1 & - \\
\hline \multicolumn{6}{|l|}{ Masarinae } \\
\hline Ceramiopsis paraguayensis Bertoni, 1921 & - & - & 7 & - & - \\
\hline Trimeria rubra Hermes \& Melo, 2006 & - & 2 & - & - & - \\
\hline Polistinae & - & - & - & - & - \\
\hline Agelaia multipicta (Haliday, 1836) & 47 & 8 & 8 & 1 & - \\
\hline Agelaia pallipes (Schrottky, 1911) & - & 2 & - & - & - \\
\hline Apoica flavissima Vecht, 1972 & 2 & - & - & - & - \\
\hline Apoica pallens (Fabricius, 1804) & - & - & - & - & 1 \\
\hline Brachygastra augusti (Saussure, 1854) & 4 & - & - & - & - \\
\hline Brachygastra lecheguana (Latreille, 1824) & 2 & 2 & 12 & 1 & 4 \\
\hline Brachygastra moulae Richards, 1978 & 13 & - & - & - & - \\
\hline Brachygastra fistulosa Naumann, 1968 & - & - & - & 4 & - \\
\hline Metapolybia sp. & - & 2 & - & - & - \\
\hline Mischocyttarus sp. 1 & 2 & - & 1 & - & 1 \\
\hline Mischocyttarus sp. 2 & 1 & - & - & - & - \\
\hline Mischocyttarus sp. 3 & 1 & - & - & - & 1 \\
\hline Mischocyttarus sp. 4 & 5 & - & 1 & 11 & - \\
\hline Mischocyttarus sp. 5 & 1 & - & 1 & - & - \\
\hline Mischocyttarus sp. 6 & 1 & - & - & - & - \\
\hline Mischocyttarus sp. 7 & 2 & - & - & - & - \\
\hline Mischocyttarus sp. 8 & 1 & - & - & - & - \\
\hline Parachartegus smithii Saussure, 1854 & - & - & - & - & 1 \\
\hline Polistes canadensis (Linnaeus, 1758) & 17 & 1 & - & 3 & - \\
\hline Polistes ferreri Saussure, 1853 & 5 & - & - & - & - \\
\hline Polistes simillimus Zikan, 1951 & 9 & 2 & - & - & - \\
\hline Polistes versicolor (Olivier, 1792) & 14 & - & 1 & - & - \\
\hline Polistes sp. 1 & - & - & - & 3 & - \\
\hline Polistes sp. 2 & - & - & - & 1 & - \\
\hline Polistes sp. 3 & - & - & 1 & - & - \\
\hline Polistes sp. 4 & 1 & - & - & - & - \\
\hline Polybia sericea (Oliver, 1796) & 14 & 7 & 5 & 7 & 6 \\
\hline Polybia ignobilis (Haliday, 1836) & 37 & 3 & 8 & 7 & 5 \\
\hline Polybia (gr. occidentalis) sp. & 33 & 7 & 36 & 6 & 3 \\
\hline Polybia paulista Ihering, 1896 & - & - & - & - & 2 \\
\hline Polybia sp. & - & - & - & 1 & - \\
\hline Protonectarina sylveirae (Saussure, 1854) & 10 & - & - & 1 & 3 \\
\hline Protopolybia exigua (Saussure, 1906) & 1 & - & - & - & 5 \\
\hline Protopolybia sedula (Saussure, 1854) & - & - & - & - & 1 \\
\hline Pseudopolybia vespiceps (Ducke, 1907) & - & - & - & 1 & - \\
\hline Synoeca cyanea (Fabricius, 1775) & - & - & - & - & 1 \\
\hline Synoeca surinama (Linnaeus, 1767) & - & 2 & - & - & - \\
\hline
\end{tabular}

maior disponibilidade e diversidade de recursos alimentares e de substratos de nidificação.

GARCETE-BARRETT (1996), em um inventário rápido na Reserva Nacional del Bosque Mbaracayú no Paraguai, registrou 28 espécies de Polistinae e 15 de Eumeninae, totalizando 43 espécies. DinIZ \& KITAYAMA (1998) levantaram na Chapada dos Guimarães 36 espécies, em 50 exemplares amostrados, indicando uma alta diversidade deste grupo para o Cerrado. HeRmes \& KöHLER (2004) identificaram 70 espécies provenientes da área do Cinturão Verde de Santa Cruz do Sul, no Rio Grande do Sul.

Se considerarmos apenas a fauna de Polistinae, verificamos que SilveIRA (2002) na Amazônia, amostrou 79 espécies em 18 gêneros, em um período de um ano, enfatizando que $2 / 3$ da diversidade das vespas sociais do Brasil está nesta região e grande parte desta fauna é endêmica. Na Bahia, SANTos et al. (2009) amostraram 19 espécies em 13 gêneros de vespas sociais, distribuídas em três fitofisionomias diferentes de Cerrado. No Mato Grosso do Sul foram registradas 37 espécies e 12 gêneros.

Apesar das metodologias de coleta nos trabalhos serem diferentes, em relação principalmente ao número de amostras e esforço amostral, a diversidade no MS foi relativamente alta em comparação com outros inventários na América do Sul. Mesmo sendo utilizados métodos não padronizados nas cinco regiões amostradas no estado, observamos uma alta beta diversidade; isto indica uma forte tendência na substituição das espécies nas diferentes assembleias de vespas que coexistem nos diferentes ecossistemas no Mato Grosso do Sul, corroborando com a ideia de que a heterogeneidade ambiental infere uma elevada diversidade de vespas (LoYolA \& MARTINS, 2008). Fatores como o estado de conservação das áreas estudadas, a disponibilidade de recursos alimentares, os locais de nidificação e a sazonalidade, podem ser 
determinantes na alta dissimilaridade entre as comunidades de vespas (SANTOS et al., 2009).

Este levantamento preliminar da fauna de Vespidae em algumas regiões do Mato Grosso do Sul, traz um número expressivo de primeiros registros de ocorrência de espécies para o estado. Isso se deve ao fato de nunca ter sido feito um inventário sistematizado nesta região. A principal coleta realizada até então foi anterior à divisão do Mato Grosso (RICHARDS, 1978); consequentemente os registros que encontramos na literatura são apenas para o MT, mesmo que possivelmente tenham sido feitas coletas em territórios que hoje pertencem ao MS.

Inventariar a assembleia de Vespidae em ecossistemas naturais resulta em uma ferramenta capaz de interpretar as condições ambientais e o estado de conservação das áreas. Estes insetos possuem características fundamentais para serem utilizados como bioindicadores em planos de manejo, por serem abundantes em inventários da entomofauna, serem de fácil identificação e por apresentarem um baixo custo de amostragem (MorATO \& CAMPOS, 2000). O gênero Agelaia, por exemplo, é amostrado exclusivamente em áreas contínuas de florestas (SILVEIRA, 2002) e pode ser um indicador expressivo da conservação das áreas cobertas por florestas no MS.

Concluímos que o Mato Grosso do Sul possui um mosaico de ecossistemas, muitas vezes integrados, distribuídos ao longo de um vasto território, proporcionando uma configuração heterogênea na estrutura da comunidade, o que reflete uma alta beta-diversidade dessas vespas e gera um imenso campo para estudos biogeográficos e ecológicos.

Agradecimentos. Agradecemos a Fundação de Apoio ao Desenvolvimento do Ensino, Ciências e Tecnologia do Estado de Mato Grosso do Sul (Fundect) e a Superintendência de Ciências e Tecnologia do Estado de Mato Grosso do Sul (Sucitec/MS) pelo convite de participação neste fascículo especial da Iheringia, Série Zoologia e o suporte financeiro para sua publicação. Agradecemos a Sérgio Ricardo Andena (Universidade Estadual de Feira e Santana - BA), Fernando Noll (UNESP), Marcel Hermes (UFPR) e Bolívar Garcete-Barrett (UFPR), pela identificação dos espécimes. A Camila Aoki (UFMS - Campus de Aquidauana), pelos dados fornecidos referentes ao Complexo Aporé-Sucuriú e Maciço do Urucum. Aos pesquisadores do Laboratório de Ecologia de Hymenoptera: Manoel Fernando Demétrio, pelas sugestões ao manuscrito e Bhrenno Maykon Trad, Felipe Varussa e Nelson Rodrigues da Silva e pelas coletas de campo. Ao $\mathrm{CNPq}$, pelo apoio financeiro. Agradecemos ao Instituto Homem Pantaneiro de Corumbá e ao ICMBio de Bonito, pela autorização de coleta.

\section{REFERÊNCIAS BIBLIOGRÁFICAS}

AbDon, M. M. \& SiLVA, J. S. V. 2006. Identificação de padrões em imagens Landsat-ETM+ para interpretação da vegetação arbórea do cerrado na sub-região de Cáceres, no Pantanal do estado do Mato Grosso. In: Semana de Geografia da Unemat, 9. (SEMAGEO), Cáceres/MT. Anais.... Cáceres, Unemat, p. 1-13.

Auko, T. H. \& Silvestre, R. 2015. First records of Plagiolabra von Schulthess (Hymenoptera: Vespidae) from Brazil. Check list 11(1):1-2.

Boggiani, P. C.; Fairchild, T. R. \& Coimbra, A. M. 1993. O grupo Corumbá (Neoproterozóico-Cambriano) na região central da Serra da Bodoquena (Faixa Paraguai) Mato Grosso do Sul. Revista Brasileira de Geociências 23:301-305.

Brothers, D. J. \& Finnamore, A. T. 1993. Superfamily Vespoidea. In: Goulet, H. \& Huber, J. T. eds. Hymenoptera of the world: an identification guide to families. Ottawa, Agriculture Canada, p. 161-278.
Bucher, H. 1980. Ecología de la fauna Chaqueña. Una revisón. Ecosur 7(4):111-159.

Carpenter, J. M. \& Garcete-Barrett, B. R. 2002. A key to the Neotropical genera of Eumeninae (Hymenoptera: Vespidae). Boletín del Museo Nacional de Historia Natural del Paraguay 14(1-2):52-73.

CArpenter, J. M. \& Marques, O. M. 2001. Contribuição ao Estudo dos Vespídeos do Brasil (Insecta, Hymenoptera, Vespoidae, Vespidae). Cruz das Almas, Universidade Federal da Bahia. (Publicações digitais, vol. 2).

Diniz, I. R. \& Kitayama, K. 1998. Seasonality of vespid species (Hymenoptera: Vespidae) in a central Brazilian Cerrado. Revista de Biología Tropical 46:109-114.

Elpino-Campos, A.; Del-Claro, K. \& Prezoto, F. 2007. Diversity of social wasps (Hymenoptera: Vespidae) in Cerrado fragments of Uberlândia, Minas Gerais State, Brazil. Neotropical Entomology 36(5):685-692.

Garcete-Barrett, B. 1996. Entomology: Hymenoptera Surveys. In: Lowen, J. et al. eds. Biological Surveys and Conservation Priorities in Eastern Paraguay. Cambridge, CBS Conservation Publications, p. $147-150$.

Gomes, B. \& Noll, F. B. 2009. Diversity of social wasps (Hymenoptera, Vespidae, Polistinae) in three fragments of semideciduous seasonal forest in the northwest of São Paulo State, Brazil. Revista Brasileira de Entomologia 53(3):428-431.

Hermes, M. \& Köhler, A. 2004. Chave ilustrada para as espécies de Vespidae (Insecta, Hymenoptera) ocorrentes no Cinturão Verde de Santa Cruz do Sul, RS, Brasil. Caderno de Pesquisa, série Biologia 16(2):65-115.

JunioR, G. M. T. \& NolL, F. 2011. Diversity of social wasps on Semideciduous Seasonal Forest fragments with different surrounding matrix in Brazil. Psyche 2011:1-8.

LOYOLA, R. D. \& Martins, R. P. 2008. Habitat structure components are effective predictors of trap-nesting Hymenoptera diversity. Basic and Applied Ecology 9:735-742.

Mechi, M. R. 1996. Levantamento da fauna de vespas na vegetação de duas áreas de Cerrado. Tese de Doutorado. São Carlos, Universidade Federal de São Carlos.

Mechi, M. R. \& Moraes, J. A. P.V. 2000. Comunidade de vespas Aculeata (Hymenoptera: Vespoidea) de uma área de cerrado e suas visitas às flores. In: SAntos, J. E. \& PIRES, J. R. S. eds. Estudos integrados em ecossistemas. Estação Ecológica de Jataí. São Carlos, RIMA, p. $765-791$.

Morato, E. F. 2004. Efeitos da sucessão florestal sobre a nidificação de vespas e abelhas solitárias. Tese de Doutorado. Belo Horizonte, Universidade Federal de Minas Gerais.

Morato, E. F. \& CAMPos, L. A. O. 2000. Efeitos da fragmentação florestal sobre vespas e abelhas solitárias em uma área da Amazônia Central. Revista Brasileira de Zoologia 17(2):429-444.

Morato, E. F.; Amarente, S. T. \& Silveira, O. T. 2008. Avaliação ecológica rápida da fauna de vespas (Hymenoptera: Aculeata) do Parque Nacional da Serra do Divisor, Acre, Brasil. Acta Amazonica 38(4):789-798.

Morrone, J. J.; Mazzucconi, S. A. \& Bachmann, A. O. 2004. Distributional patterns of Chacoan water bugs (Heteroptera: Belostomatidae, Corixidae, Micronectidae and Gerridae). Hydrobiologia 523:159-173.

Noguchi, D. K.; Nunes, G. P. \& Sartori, A. L. B. 2009. Florística e síndromes de dispersão de espécies arbóreas em remanescentes de Chaco de Porto Murtinho, Mato Grosso do Sul, Brasil. Rodriguésia 60(2):353-365.

O'DonNell, S. 1995. Necrophagy by Neotropical swarm-founding wasps. (Hymenoptera: Vespidae: Epiponini). Biotropica 27(1):133-136.

Pagotto, C. S. \& Souza, P. R. 2006. Biodiversidade do Complexo Aporé-Sucuriú: subsídios à conservação e ao Manejo do cerrado: área prioritária 316-Jauru /Orgs. Campo Grande, Mato Grosso do Sul, Ed. UFMS. 308p.

Pickett, K. M. \& Carpenter, J. M. 2010. Simultaneous analysis and the origin of Eusociality in the Vespidae (Insecta: Hymenoptera). Arthropod Systematics \& Phylogeny 61(1):3-33.

Potт, A. \& Potт, V. J. 2003. Espécies de fragmentos florestais em Mato Grosso do Sul. In: CostA, R. B. org. Fragmentação florestal e alternativas de desenvolvimento rural na região centro-oeste. Campo Grande, UCDB, p. 26-52. 
Prado, D. E. \& GibBs, P. E. 1993. Patterns of species distribution in the dry seasonal forest of South America. Annals of the Missouri Botanical Garden 80:902-927.

Rasmussen, C. \& Asenjo, A. 2009. Checklist to the wasps of Peru (Hymenoptera, Aculeata). Zookeys 15:1-78.

RAw, A. 1998a. Population densities and biomass of neotropical social wasps (Hymenoptera, Vespidae) related to colony size, hunting range and wasps size. Revista Brasileira de Zoologia 15:815-822.

RAw, A. 1998b. Social wasps (Hymenoptera, Vespidae) of the Ilha de Maracá. In: W. Milliken \& J.A. Ratter, eds. Maracá: The biodiversity and environment of Amazonian Rainforest. Chichester, John Wiley \& Sons, p.307-321.

Richards, O. W. 1978. The social wasps of the Americas excluding the Vespinae. London, British Museum (Natural History). 296p.

SAntos, B. B. 1996. Ocorrência de vespídeos sociais (Hymenoptera, Vespidae) em pomar em Goiânia, Goiás, Brasil. Agrárias 15:43-46.

Santos, G. M. M.; Bicharra Filho, C. C.; Resende, J. J.; Cruz, J. D. DA \& MARques, O. M. 2007. Diversity and community structure of social wasps (Hymenoptera, Vespidae) in three ecosystems in Itaparica Island, Bahia State, Brazil. Neotropical Entomology 36:180-185.

Santos, G. M. M.; Cruz, J. D.; Marques, O. T. \& Gobbi, N. 2009. Diversidade de vespas sociais (Hymenoptera: Vespidae) em áreas de Cerrado na Bahia. Neotropical Entomology 38(3):317-320.

SARmiento, C. 1997. Véspidos de Colombia (Hymenoptera: Vespidae). Tesis de Maestría. Bogotá, Universidad Nacional de Colombia, Instituto de Ciencias Naturales.

SARMiento, C. 1999. Los véspidos solitarios de Colombia (Hymenoptera: Vespidae: Eumeninae, Masarinae). In: Amat, G.; Andrade, C. G. \& FERnández, F. eds. Insectos de Colombia. Academia Colombiana de Ciencias Exactas, Físicas y Naturales, Santa Fé de Bogotá. vol. 1, p. 53-105

SARmiento, C. E \& CARpenter, J. M. 2006. Familia Vespidae. In: Fernández, F. \& Sharkey, M. J. eds. Introducción a los Hymenoptera de la Región Neotropical. Bogotá, Sociedad Colombiana de Entomologia y Universidad Nacional de Colombia, p. 539-555.

SiLvA, S. 2007. Vespas sociais da Floresta Nacional de Caxiuanã, Melgaço, Pará. Descrição da fauna numa grade de $25 \mathrm{~km}^{2}$ e comparação entre protocolos de amostragem (Hymenoptera, Vespidae). Dissertação de Mestrado. Belém, Museu Paraense Emílio Goeldi.

Silva, S. S. \& Silveira, O. T. 2009. Vespas sociais (Hymenoptera, Vespidae, Polistinae) de floresta pluvial Amazônica de terra firme em Caxiuanã, Melgaço, Pará. Iheringia, Série Zoologia 99(3):317-323.

SiLveIrA, O. T. 2002. Surveying Neotropical social wasps. An evaluation of methods in the "Ferreira Penna" Research Station (ECFPn), in Caxiuanã, PA, Brazil (Hym., Vespidae, Polistinae). Papéis Avulsos de Zoologia 42(12):299-323.

Silveira, O. T.; Costa Neto, S. V. \& Silveira, O. F. M. 2008. Social wasps of two wetland ecosystems in Brazilian Amazonia (Hymenoptera, Vespidae, Polistinae). Acta Amazonica 38(2):333-344.

Silvestre, R.; Demétrio, M. F. \& Delabie, J. H. C. 2012. Community structure of leaf-litter ants in a Neotropical dry forest: A Biogeographic approach to explain betadiversity. Psyche 2012:1-15.

Somavila, A.; KöHler, A. \& Hermes, M. G. 2010. Contribuição aos estudos dos Vespidae ocorrentes no estado do Rio Grande do Sul (Insecta, Hymenoptera). Revista Brasileira de Biociências 8(3):257-263.

Souza, M. B.; Silveira, M.; Lopes, M. R. M.; Vieira, L. J. S.; Guilherme, E.; Calouro, A. M. \& Morato, E. F. 2003. A Biodiversidade no Estado do Acre: Conhecimento Atual, Conservação e Perspectivas. T \& C Amazônia 1(3):45-56.

Togni, O. C. 2009. Diversidade de vespas sociais (Hymenoptera: Vespidae) na Mata Atlântica do litoral norte do Estado de São Paulo. Dissertação de Mestrado. São Carlos, Universidade Estadual Paulista.

VAnZOLINI, P. E. 1988. Distributional patterns of South American lizards. In: Vanzolini, P. E. \& Heyer, W.R. eds. Proceedings of a Workshop on Neotropical Distribution Patterns. Rio de Janeiro, Academia Brasileira de Ciências, p. 317-342.

Willink, A. \& Roig-Alsina, A. 1998. Revision del genero Pachodynerus Saussure (Hymenoptera: Vepidae, Eumeninae). Contributions of the American Entomological Institute 30(5):1-117.

Zanella, F. C. V. 2011. Evolução da Biota da Diagonal de Formações Abertas Secas da América do Sul. In: Carvalho, C. J. B. \& Almeida, E.A.B. eds. Biogeografia da América do Sul: padrões e processos. São Paulo, Editora Roca, p. 198-220. 